\title{
Unusual behavior of the magnetization reversal in soft/hard multisegmented nanowires
}

\author{
Rosa M. Corona ${ }^{1}$, Ali C. Basaran ${ }^{2}$, Juan Escrig ${ }^{1,3}$, and Dora Altbir ${ }^{1,3}$ \\ ${ }^{1}$ Departamento de Física, Universidad de Santiago de Chile (USACH), Av. Ecuador 3493, 9170124 Santiago, Chile \\ ${ }^{2}$ Deparment of Physics and Center for Advanced Nanoscience, \\ University of California San Diego, La Jolla, California 92093, USA \\ ${ }^{3}$ Center for the Development of Nanoscience and Nanotechnology (CEDENNA), 917-0124 Santiago, Chile
}

\begin{abstract}
The magnetization reversal mechanisms in soft/hard multisegmented nanowires have been investigated using numerical simulations. In most of the studied systems the magnetization reversal process starts at the ends. However in short soft/hard/soft nanowires, the magnetization reversal process starts at the center of the wire, generating an unusual behavior of the coercivity as a function of the length.
\end{abstract}

\section{INTRODUCTION}

During the last decades, the study of magnetic nanowires $(\mathrm{NW})$ has attracted strong attention because of their potential for applications in diverse areas such as binary information, high-density and high-speed data storage [1, 2], and biology [3]. Different lithographic techniques can be used to grow magnetic NWs, however they are usually very expensive and time consuming. An alternative is the use of chemical template-based methods combined with high-yield electrochemical deposition techniques. A widespread example are anodic aluminum oxide (AAO) membranes, that allow a precise control of the geometrical features, such as nanopore diameter, length, and periodicity, by simply changing the anodization conditions [4-6]. Hence, several studies concerning single-element magnetic NW arrays of $\mathrm{Ni}, \mathrm{Co}, \mathrm{Fe}$, and their alloys can be found in the literature [7-14].

Nanowires are usually very long, with an aspect ratio (length/diameter) larger than 10 that allows such structures to exhibit a significant shape anisotropy. Because of this, a single NW exhibits two lower energy states in which most of the magnetic moments are parallel to the wire axis, with square hysteresis loops characteristics of this bistable behavior [15]. However, in an array, interelement interactions and its distributions generate more complex reversal processes with non-square hysteresis cycles $[16,17]$.

Using similar techniques to those previously describes, it is possible to produce multisegmented nanowires, that is, wires composed by layers of different materials [18]. For example, Kuo Qi et al. [19] prepared $\mathrm{NiCoCu} / \mathrm{Cu}$ multisegmented nanowires using electrodeposition techniques and dc current pulses. Almasi-Kashi et al. [20] were able to control the thickness of each segmentd by varying the number of ac-pulses in each layer of $\mathrm{Fe} / \mathrm{Cu}$ nanowires. Tang et al. [21] studied $\mathrm{CoNi} / \mathrm{Cu}$ multisegmented nanowires and observe that the interaction among magnetic segments defines the magnetization reversal. This was confirmed in a recent paper by Rando et al. [22], who studied the reversal modes in arrays of long multisegmented $\mathrm{Ni} / \mathrm{Cu}$ wires. Finally, Pereira et al. [23] introduced a new soft/hard nanostructure based on multisegmented CoNi nanowire arrays.

An interesting system is formed by wires with soft and hard segments, since in such structures coercivity is usually determined by the hard phase while the remanence is originated in the soft phase [24-27], generating a mix of properties that can be tailored with the adequate election of materials. An example is shown in a paper by Forster et al. [28], who made micromagnetic calculations on magnetic wires composed of two parts, a soft magnetic part and hard magnetic part, showed that the domain wall velocity increases with increasing wire diameter and decreasing damping constant.

Agramunt-Puig et al. [29] used a simple but powerful model to study the collective magnetic response of large arrays of segmented nanowires comprising two magnetic segments of different coercivity separated by a nonmagnetic spacer. The authors showed that the geometry can be optimize to attain antiparallel or parallel magnetic alignment, as required in magnetic sensors based on the GMR effect and other applications. A similar system was addressed by Allende et al. [30] who investigated the effect of the dipolar magnetic interaction between magnetic layers mediated by non-magnetic ones in segmented nanowires. The dipolar interaction generates two different reversal modes, according to its strength; an independent route, in which the segments reverse simultaneously, and a dependent one, when the segments are strongly coupled. This mode exhibits larger coercivities as compared with the ones that show parallel reversal mode and could be useful for the creation of multistable systems. Gapin et al. [31] proposed the use of soft/hard composite nanowire arrays of $\mathrm{Ni} / \mathrm{CoPt}$ for patterned media, and S. M. Hamidi et al. [27] synthesized Ni, Co and $\mathrm{Ni} / \mathrm{Co}$ multisegmented nanowires from a combined technique of AAO template and electrodeposition method, and showed that the multisegmented $\mathrm{Ni} / \mathrm{Co}$ nanowires can be used as efficient magnetic field sensor.

The systems mentioned above have a strong shape anisotropy due to their length, which defines their magnetic behavior. However, the study of wires of reduced length has been less frequent, even more if they are formed by alternate segments of soft and hard materials. For this reason, in this work we focus on short wires 
made up of Co and Ni segments, and compare their reversal modes with those of long wires. Our results show different reversal modes as a function of the length, evidencing an unusual behavior of coercivity in short multisegmented nanowires.

\section{MICROMAGNETIC SIMULATION}

We have studied the hysteresis curves and magnetization reversal mechanisms for non-interacting nanowires using a micromagnetic simulator [32]. However, our results are also valid for arrays with inter-element distance large enough to consider the nanowires as noninteracting. As stated above, we focus on nanowires with a fix diameter of $d=70 \mathrm{~nm}$ and a total length, $L$, varying between 150 and $1500 \mathrm{~nm}$ (see schematic drawings inserted in Fig. 1). The multisegmented nanowires are composed of three segments, each with a length $L / 3$, where the magnetically soft (hard) material is sandwiched by the other two hard (soft) materials.

Moreover, the magnetization dynamics in OOMMF is governed by the Landau-Lifshitz-Gilbert equation (LLG)

$$
\frac{d \vec{M}}{d t}=-\gamma \vec{M} \times \vec{H}_{\mathrm{eff}}+\frac{\alpha}{M_{s}} \vec{M} \times \frac{d \vec{M}}{d t},
$$

where $\gamma$ is the gyromagnetic ratio of the free electron spin and $\alpha$ is a phenomenological damping constant. The equation describes both the precession and relaxation motion of the magnetization in an effective field $\vec{H}_{\text {eff }}=\partial G / \partial \vec{M}$, where $G$ is the Gibbs free energy that considers the exchange, dipolar and Zeeman contributions. We used exchange constants $A^{N i}=9 \times 10^{-12} \mathrm{~J} / \mathrm{m}$ and $A^{C o}=30 \times 10^{-12} \mathrm{~J} / \mathrm{m}$. The saturated magnetizations for $\mathrm{Ni}$ and Co were $M_{s}^{N i}=480 \times 10^{3} \mathrm{~A} / \mathrm{m}$ and $M_{s}^{C o}=1400 \times 10^{3} \mathrm{~A} / \mathrm{m}$, respectively. Finally, an external field of $H_{z}=1 \mathrm{~T}$ was applied with $\Delta H_{z}=0.002 \mathrm{~T}$ steps.

At this point it is important to note that the interface is located exactly between cells of different materials. For our calculations we have used the Oxs_ExchangePtwise formulation proposed in the OOMMF [32] that points out that the exchange energy density at a cell $i$ is computed across its nearest six neighbors, $\mathrm{Ni}$, using the formula

$$
E_{i}=\sum_{i \in \mathrm{Ni}} A_{i j, \mathrm{eff}} \frac{m_{i} \cdot\left(m_{i}-m_{j}\right)}{\Delta_{i j}^{2}},
$$

where $\Delta_{i j}^{2}$ is the discretization step size from cell $i$ to cell $j$ in meters, and

$$
A_{i j, e f f}=\frac{2 A_{i} A_{j}}{A_{i}+A_{j}}
$$

in this way interfaces of different materials can be considered. Considering that the magnetocrystalline anisotropy of the samples can vary as a function of the geometric parameters and of the synthesis process of the wires [33], for simplicity we will not consider this energetic term, something also valid for polycrystalline samples. For our simulations we consider cell sizes of $2.5 \times 2.5 \times 2.5 \mathrm{~nm}^{3}$, this mesh is small enough to adequately describe systems with cylindrical symmetry, as shown in Refs. [34, 35]. In all the simulations we considered a damping constant equal to 0.5 .

\section{RESULTS AND DISCUSSION}

We start by studying the role of the length in wires with different magnetic structures. Figure 1 shows the hysteresis curves of soft $(\mathrm{Ni})$, hard (Co) and multisegmented soft/hard/soft and hard/soft/hard nanowires with $L=150$ (left column) and $L=750 \mathrm{~nm}$ (right column). In this figure we observe that nanowires with $L=750 \mathrm{~nm}$ exhibit an almost bistable behavior, because the reversal starts by the nucleation of domain walls at their ends, that propagate towards the center of the wire. Even more, in Figs. 1e $-1 \mathrm{~h}$, all with an aspect ratio $L / d=10.7$, the shape of the hysteresis curves is almost the same, indicating that for long aspect ratios the dominant contribution to the reversal process comes from the shape anisotropy.

A different situation occurs in short nanowires $(L=$ $150 \mathrm{~nm}, L / d=2.14)$. In this case, since the shape anisotropy is weak, the lower energy configuration is not a full ferromagnetic state along the wire axis [36] as in the previous case, generating a different reversal process and non-rectangular hysteresis loops, as will be explained in detail below. In this case, important differences appear in the hysteresis curves, depending on the material configuration, as shown in Figs. 1a - 1d.

In figure 2 we summarize our results for the coercivity (a) and remanence (b) for different lengths of the nanowires, showing the strong impact of the geometry. Two well-defined behaviors for coercivity and remanence are evidenced in this figure. The first one involves nanowires with $L \leq 700 \mathrm{~nm}$ (short wires, with $L / d \leq 10$ ), while the other one is evidenced for nanowires with $L>700 \mathrm{~nm}$ (long wires, with $L / d>10$ ). The fluctuations in coercivity for short wires are due to the fact that the segments making up the wire have a length comparable to the width of the domain walls that are formed. Therefore, as the length of the wires increases, the size of the domain wall varies, leading to a variation in the coercivity. In particular, for long nanowires, both coercivity and remanence exhibit an almost constant value as a function of length. If we have a hard material at the ends of the wire, the coercivity is larger and the remanence is lower than if we have the opposite situation, that is, soft materials at the ends of the wire, regardless of the number of segments of the nanostructure. On the other hand, for short nanowires, in general the coercivity decreases as we increase the length, except when we have a multisegmented soft/hard/soft nanostructure. We will 


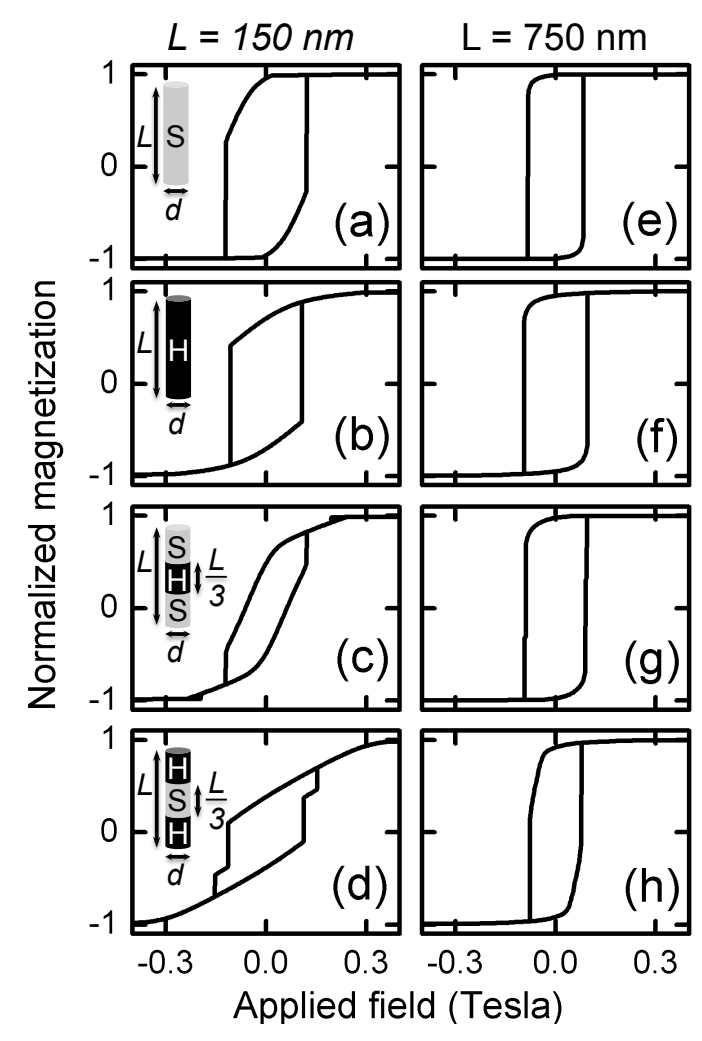

FIG. 1. (Color online) Hysteresis curves of soft (a and e), hard (b and f) and multisegmented soft/hard/soft (c and g) and hard/soft/hard (d and h) nanowires with $L=150$ (left column) and $L=750 \mathrm{~nm}$ (right column).

see later that this unusual behavior is due to a change in the magnetization reversal mode of the wire for this particular case. Finally, we observe that for these short nanowires, remanence increases as we increase the length of the wire.

To understand in more detail this behavior, we analyze the magnetization reversal processes of multisegmented nanowires with $L=150 \mathrm{~nm}$ (see Fig. 3) and $L=750 \mathrm{~nm}$ (see Fig. 4), obtaining snapshots of the magnetization at specific moments in the hysteresis cycle. The nucleation and propagation of domain walls (if any) is monitored by the value $m_{i}(z)=M_{i}(z) / M_{s}(z)$, the average value of the component of the magnetic moment, relative to the saturation value, at position $z$. In Figs. 3 and 4, dotted (green) lines represent the average axial component of the magnetization, $m_{z}$, while the average of the radial component, $m_{\rho}$, is illustrate by solid (red) lines. For all the cases studied the radial component of the magnetization is zero.

Figure 3a illustrates our results for a multisegmented soft/hard/soft nanowire with $L=150 \mathrm{~nm}$. We start our simulations with the wire fully saturated along the $z$ axis, with $m_{z}=1$ (red) under an external field of $10 \mathrm{kOe}$. In a soft/hard/soft structure, while decreasing the field, the reversal process stars by reversing the magnetization at
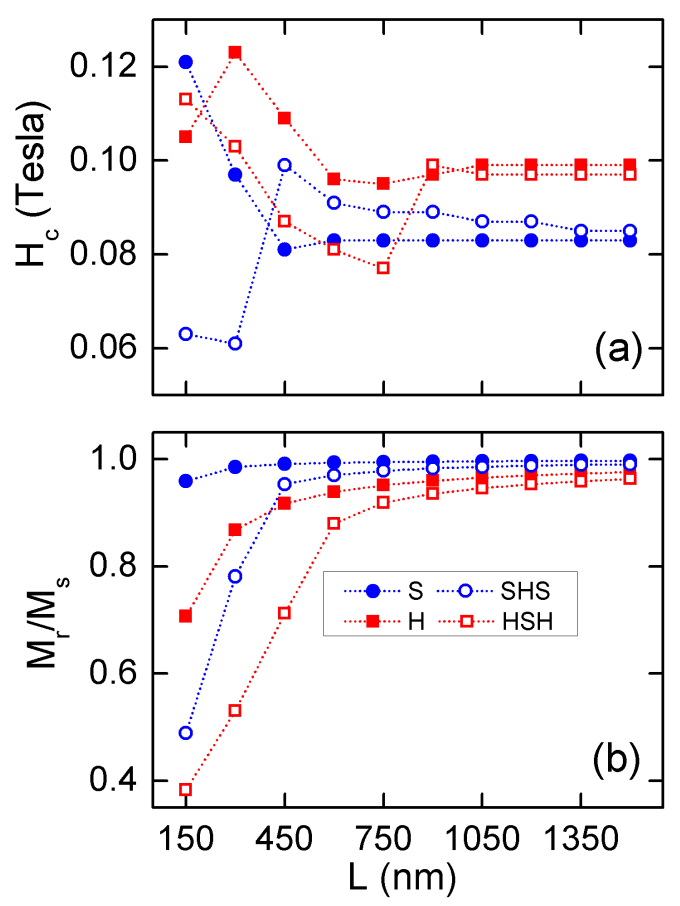

FIG. 2. (Color online)(a) Coercivity and (b) remanence of soft (filled blue circles), hard (filled red squares) and multisegmented soft/hard/soft (hollow blue circles) and hard/soft/hard (hollow red squares) nanowires for different length values.

the center of the wire, as shown in Figs. 3a, snapshot (i). This unexpected behavior continues by reversing the magnetization of the soft ends and then all the surface, leading a central section of hard material unreversed, as shown in the color figure accompanying snapshot (iii). The magnetization of the segments at the ends is faster than the reversal of the central segment, finishing the reversal of the extremes, while the central segment has not yet reverted (iv). It is interesting to mention that among all nanowires with all lengths considered, this is the only case in which the system stars reversing its magnetization at the center of the wire, producing the unusual behavior of the coercivity illustrated in Fig 2a.

On the other hand, from Fig. 3b we can observe that the multisegmented hard/soft/hard nanowire stars reversing its magnetization at the hard segments of the wire ends (i). This process continues until the segments of the ends have formed a perfect vortex (ii). In this case, and considering the length of the nanostructure, no nucleation and propagation of domain walls is observed. Then, an abrupt change of the magnetization of the central segment occurs, which is visualized in the hysteresis curve as an abrupt Barkhausen jump (iv), after which the reversal continues until the full reversion. Since along all the process $m_{\rho}=0$ some kind of vortex structure must appears during the reversal.

It is interesting to observe in Fig. 4 that for $L=750$ 


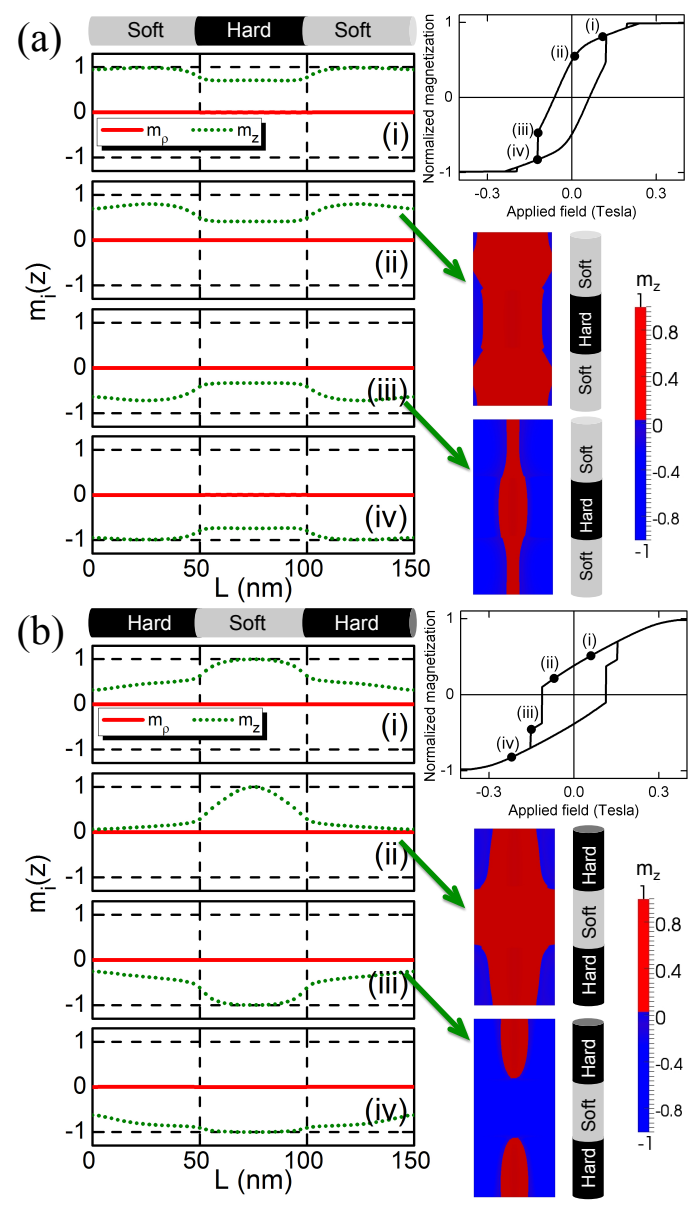

FIG. 3. (Color online) Right side represents the average value of the component of the magnetic moment at the position $z$ relative to the saturation value for $L=150 \mathrm{~nm}$. The abscissa represents the axial coordinate, $z$, along the multisegmented soft/hard/soft (a) and hard/soft/hard (b) nanowires and the ordinates give the average components of the magnetization. $m_{z}$ is denoted in dotted (green) lines and $m_{\rho}$ is depicted in solid (red) lines. Left side represents the hysteresis cycle and the reversal process in a color scale.

$\mathrm{nm}$, both multisegmented wires reverse their magnetization by similar mechanisms. In both cases the magnetization at the center of the nanostructure remains pinned parallel to its axis, regardless of whether this segment is magnetically soft or hard. In addition, it is important to mention that in both cases the magnetization reversal starts at the ends (i), nucleating a vortex that grows until occupying the entire segment (iii). Once the segments have formed vortices, the magnetization of the central segment starts reversing, dragging with it the magnetization of the ends (iv). This behavior is similar to what is described by Ivanov et al. [38], in our case in spite we have neglected anisotropy, the DW pinning comes from the different values of the exchange constant.

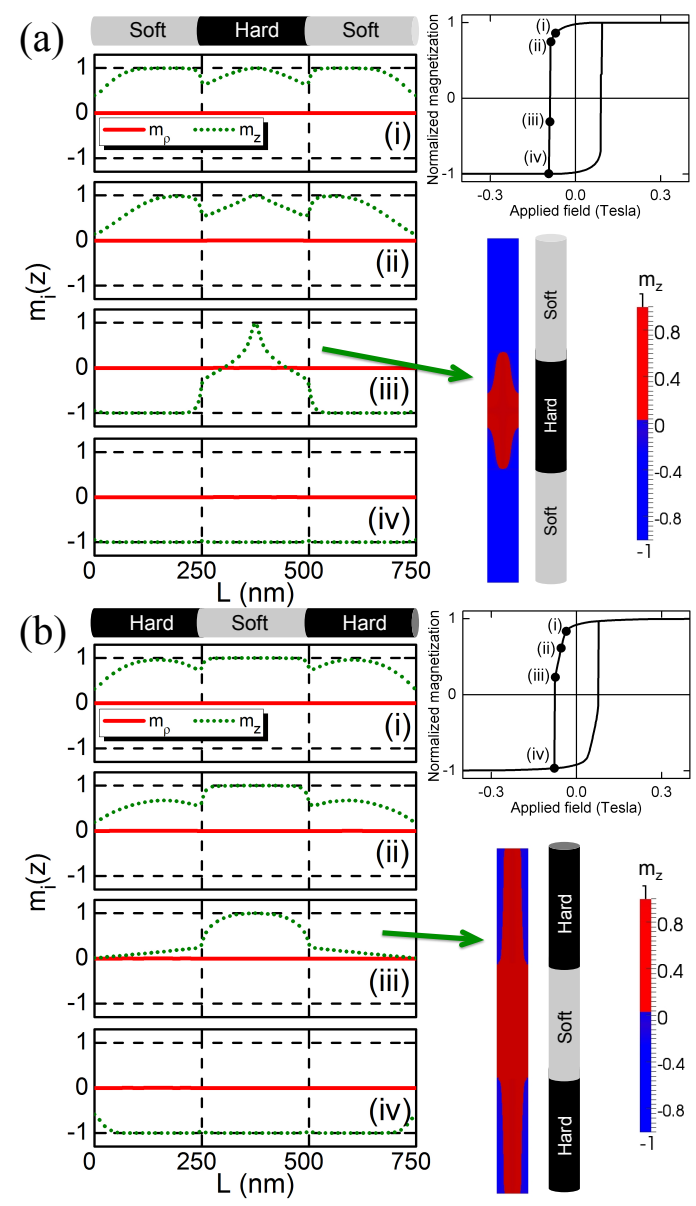

FIG. 4. (Color online) Right side represents the average value of the component of the magnetic moment at the position $z$ relative to the saturation value for $L=750 \mathrm{~nm}$. The abscissa represents the axial coordinate, $z$, along the multisegmented soft/hard/soft (a) and hard/soft/hard (b) nanowires and the ordinates give the average components of the magnetization. $m_{z}$ is denoted in dotted (green) lines, and $m_{\rho}$ is depicted in solid (red) lines. Left side represents the hysteresis cycle and the reversal process in a color scale.

\section{CONCLUSIONS}

We have numerically investigated the hysteresis loops for non-interacting magnetically soft $(\mathrm{Ni})$, hard (Co) and multisegmented (soft/hard/soft and hard/soft/hard) nanowires as a function of their length. For all the cases studied it is obtained that the radial component of the magnetization is zero along the process, indicating that the reversal process of the magnetization considers the formation of some kind of vortices structures. The results presented above show that there are two welldefined behaviors, one for long nanowires with $L / d>10$, where both coercivity and remanence exhibit almost constant behavior as a function of length, and other for short nanowires with $L / d \leq 10$. For the latter case, in general the coercivity decreases as we increase the 
length of the wire, except when we have a multisegmented soft/hard/soft nanostructure. The latter is the only case in which the system starts reversing its magnetization from the center of the nanostructure. Finally we observe that for short nanowires, remanence increases as we increase the wire length. These results let to understand the behavior of the magnetization in nanowires as a function of their geometric and magnetic parameters, allowing to taylor such characteristics for specific purposes.

\section{ACKNOWLEDGMENTS}

We thank Ivan K. Schuller for his insightful comments. This was a very interactive research. The design of the problem and writing of the manuscript was done jointly between all the authors. In Chile, the authors acknowledge financial support from the Fondecyt Grants 1150952 and 1120356, Conicyt Ph.D. Program Fellowships, Proyecto Basal USA 1555 and Dicyt-USACH (Programa Apoyo a la Investigación), and the Financiamiento Basal para Centros Cientícos y Tecnológicos de Excelencia, under project FB0807. ACB helped guide the simulations and discussed on a daily basis the numerical results and was funded while at UCSD by DOE grant number FG02-87ER-45332.

\section{REFERENCES}

[1] R. Hertel, Journal of Physics: Condensed Matter 28, 48 (2016).

[2] Y. Zhang, X. Zhang, J. Hu, Z. Zheng, Z. Zhang, N. Vernier, D. Ravelosona, W. Zhao, Scientific Reports 6, 35062 (2016).

[3] Warren C. W. Chan, Bio-Applications of Nanoparticles, Landes Bioscience and Springer Science+Business Media, New York (2007).

[4] H. Masuda, K. Fakuda, Science 268, 1466 (1995).

[5] M. Vazquez, M. Hernandez-Velez, A. Asenjo, D. Navas, V. M. Prida, K. Pirota, O. Sanchez, J. L. Baldonedo, Physica B 384, 36 (2006).

[6] R. Lavin, J. C. Denardin, A. P. Espejo, A. Cortés, H. Gómez, J. Appl. Phys. 107, 09B504 (2010).

[7] L. G. Vivas, M. Vazquez, J. Escrig, S. Allende, D. Altbir, D. C. Leitao, J. P. Araujo, Phys. Rev. B 85, 035439 (2012).

[8] M. P. Proenca, C. T. Sousa, J. Escrig, J. Ventura, M. Vazquez, J. P. Araujo, J. Appl. Phys. 113, 093907 (2013).

[9] A. Pereira, C. Gallardo, A. P. Espejo, J. Briones, L. G. Vivas, M. Vazquez, J. C. Denardin, J. Escrig, J. Nanopart. Res. 15, 2041 (2013).

[10] M. S. Arshad, S. Sturm, J. Zavasnik, A. P. Espejo, J. Escrig, M. Komelj, P. J. McGuiness, S. Kobe, K. Z. Rozman, J. Nanopart. Res. 16, 2688 (2014).

[11] D. Li, S. J. Li, Y. T. Zhou, Y. Bai, Y. L. Zhu, W. J. Ren, G. Long, H. Zeng, Z. D. Zhang, J. Appl. Phys. 117, 17E702 (2015).

[12] J. Garcia, V. M. Prida, L. G. Vivas, B. Hernando, E. D. Barriga-Castro, R. Mendoza-Resendez, C. Luna, J. Escrig, M. Vazquez, J. Mater. Chem. C 3, 4688 (2015).

[13] A. I. Vorobjova, D. L. Shimanovich, K. I. Yanushkevich, S. L. Prischepa, E. A. Outkina, Nanotechnology 7, 17091717 (2016).

[14] A. H. Montazer, A. Ramazani, M. A. Kashi, J. Zavanik, J. Phys. D: Appl. Phys. 49, 445001 (2016).

[15] S. Castillo-Sepúlveda, R. M. Corona, D. Altbir, J. Escrig, J. Magn. Magn. Mater. 416, 325-328 (2016).

[16] F. Zighem, T. Maurer, F. Ott, G. Chaboussant, J. Appl. Phys. 109, 013910 (2011).

[17] D. Salazar-Aravena, R. M. Corona, D. Goerlitz, K. Nielsch, J. Escrig, J. Magn. Magn. Mater. 346, 171-174 (2013).
[18] B. Leighton, O. J. Suarez, P. Landeros, J. Escrig, Nanotechnology 20, 385703 (2009).

[19] K. Qi, X. Li, H. Zhang, L. Wang, D. Xue, H. Zhang, B. Zhou, N. J. Mellors, Y. Peng, Nanotechnology 23, 505707-505713 (2012).

[20] M. Almasi-Kashi, A. Ramazani, F. Kheyri, E. JafariKhamse, Mater. Chem. Phys. 144, 230-234 (2014).

[21] X.-T. Tang, G.-C. Wang, M. Shima, J. Magn. Magn. Mater. 309, 188-196 (2007).

[22] E. A. Rando, S. Allende, J. Appl. Phys. 118, 013905 (2015).

[23] A. Pereira, J. L. Palma, M. Vazquez, J. C. Denardin, J. Escrig, Phys. Chem. Chem. Phys. 17, 5033 (2015).

[24] N. L. Guo, G. P. Zhao, H. W. Zhang, X. L. Zhou, Y. Deng, J. Magn. Magn. Mater. 323, 3049-3053 (2011).

[25] W. Zhang, G. P. Zhao, X. H. Yuan, L. N. Ye, J. Magn. Magn. Mater. 324, 4231-4236 (2012).

[26] E. Jafari-Khamse, M. Almasi Kashi, A. Ramazani, Current Appl. Phys. 16, 486-496 (2016).

[27] S. M. Hamidi, B. Mosaeii, M. Afshamia, A. Aftabi, J. Appl. Phys. 417, 413-419 (2016).

[28] H. Forster, T. Schrefl, W. Scholz, D. Suess, V. Tsiantos, J. Fidler, J. Magn. Magn. Mater. 249, 181-186 (2002).

[29] S. Agramunt-Puig, N. Del-Valle, E. Pellicer, J. Zhang, J. Nogués, C. Navau, A. Sanchez, J. Sort, New J. Phys. 18, 013026 (2016).

[30] S. Allende, N. M. Vargas, D. Altbir, V. Vega, D. Gorlitz, K. Nielsch, Appl. Phys. Lett. 101, 122412 (2012).

[31] A. I. Gapin, X. -R. Ye, L. -H Chen, D. Hong, S. Jin, IEEE Trans. Magn. 43, 2151 (2007).

[32] M. J. Donahue, D. G. Porter, OOMMF User's Guide, Version 1.2a3 http://math.nist.gov/oommf (2002).

[33] L. V. Vivas, J. Escrig, D. G. Trabada, G. A. BadiniConfalonieri, M. Vázquez, Appl. Phys. Lett. 100, 252405 (2012).

[34] X. Han, Q. Liu, J. Wang, S. Li, Y. Ren, R. Liu, F. Li, J. Phys. D: Appl. Phys. 42, 095005 (2009).

[35] M. Chandra Sekhar, H. F. Liew, I. Purnama, W. S. Lew, G. C. Han, Appl. Phys. Lett. 101, 152406 (2012).

[36] P. Landeros, J. Escrig, D. Altbir, D. Laroze, J. d'Albuquerque e Castro, P. Vargas, Phys. Rev. B 71, 094435 (2005). 
[37] Y. P. Ivanov, M. Vázquez, O. Chubykalo-Fesenko, J. Phys. D: Appl. Phys. 46, 485001 (2013).

[38] Y. P. Ivanov, A. Chuvilin, S. Lopatin, J. Kosel, Acs Nano 10, 5326 (2016). 